\title{
Selective downregulation of distinct circRNAs in the tissues and plasma of patients with primary hepatic carcinoma
}

\author{
YAN DING $^{1,2^{*}}$, ANNING FANG $^{3 *}$, JIALAI YAN $^{4}$, JIE DUAN $^{1}$, \\ NIANYUE WANG ${ }^{5}$, YONGXIANG YI ${ }^{1}$ and CHUANLAI SHEN ${ }^{2}$ \\ ${ }^{1}$ Department of Hepatobiliary Surgery, The Second Hospital of Nanjing, Nanjing, Jiangsu 210003; \\ ${ }^{2}$ Department of Microbiology and Immunology, Medical School, Southeast University, Nanjing, Jiangsu 210009; \\ Departments of ${ }^{3}$ Basic Medicine and ${ }^{4}$ Medical Technology, Anhui Medical College, Hefei, Anhui 230601; \\ ${ }^{5}$ Department of Clinical Laboratory, The Second Hospital of Nanjing, Nanjing, Jiangsu 210003, P.R. China
}

Received April 13, 2019; Accepted September 6, 2019

DOI: $10.3892 / \mathrm{ol} .2019 .10908$

\begin{abstract}
Multiple studies have indicated that circular RNAs (circRNAs) are closely associated with malignant tumor development and metastasis. However, the significance of circRNAs in primary hepatic carcinoma (PHC), particularly in the plasma, remains largely undetermined. In the current study, circRNA expression profiles in three pairs of tumor and adjacent normal samples from patients with PHC, were examined using circRNA chip screening. A total of 80 circRNAs were upregulated, while 75 circRNAs were downregulated in PHC tissues, relative to para-tumor tissues (fold change, 21.5 ). A total of two upregulated circRNAs and three downregulated circRNAs were selected as candidates for further validation of their differential expression. This was performed using reverse transcription-quantitative PCR with 11 pairs of PHC tissues and para-tumor tissues. The results indicated that hsa_circ_0003056 exhibited reduced expression in PHC tissues. Moreover, hsa_circ_0003056 and hsa_circ_0067127 were quantified in the plasma samples of
\end{abstract}

Correspondence to: Professor Chuanlai Shen, Department of Microbiology and Immunology, Medical School, Southeast University, 87 Dingjiaqiao Road, Nanjing, Jiangsu 210009, P.R. China

E-mail: chuanlaishen@seu.edu.cn

Dr Yongxiang Yi, Department of Hepatobiliary Surgery, The Second Hospital of Nanjing, 1-1 Zhongfu Road, Nanjing, Jiangsu 210003, P.R. China

E-mail: ian0126@163.com

*Contributed equally

Abbreviations: circRNAs, circular RNAs; PHC, Primary hepatic carcinoma; HCC, Hepatocellular carcinoma; RT-qPCR, reverse transcription-quantitative PCR; miRNAs, microRNAs; GO, Gene ontology; KEGG, Kyoto Encyclopedia of Genes and Genomes; ncRNA, non-coding RNA; DE, differential expression

Key words: circRNAs, primary hepatic carcinoma, circular RNA chip screening
35 PHC patients and 32 healthy donors. The results revealed that hsa_circ_0067127 was significantly downregulated in the patients' plasma. Finally, a competing endogenous RNA network was constructed, which consisted of one circRNA (hsa_circ_0003056 or has_circ_0067127), five miRNAs and miRNA-targeted genes (mRNAs). Kyoto Encyclopedia of Genes and Genomes pathway analysis indicated that differentially expressed (DE) genes were significantly enriched in the pathway associated with 'regulation of the pluripotency of stem cells' for hsa_circ_0003056, and 'ubiquitin-mediated proteolysis' and 'prostate cancer' for hsa_circ_0067127. Gene ontology analysis revealed that DE genes were primarily associated with the 'modulation of kinase activity' and 'intracellular and transmembrane-ephrin receptor activity' for hsa_circ_0003056, 'artery morphogenesis activity', 'HOPS complex and transferase activity' and in 'transferring acyl groups' for hsa_circ_0067127. This approach indicated that hsa_circ_0003056 in PHC tissue, and hsa_circ_0067127 in PHC plasma, are downregulated and may be implicated in the tumorigenesis of PHC.

\section{Introduction}

Primary hepatic carcinoma (PHC) is one of the most common malignancies in China (1). An estimated 466,000 new PHC cases, and 422,000 PHC-associated mortalities occurred in China in 2015 , with only $10 \%$ of patients surviving $>5$ years (2). Furthermore, due to the lack of sensitive markers for early diagnosis, only $10-20 \%$ of patients with $\mathrm{PHC}$ are suitable for surgical resection, local ablation and other potential therapies (3). The current biomarkers that are used for the auxiliary diagnosis of PHC include alpha fetoprotein (AFP), AFP-L3 isoform ratio, glypican-3, des- $\gamma$-carboxy-prothrombin, golgi protein 73 and alpha L-fucus glycosidase. However, these markers, even when utilized together, are not sufficient for the identification or early diagnosis of PHC. It is therefore necessary to identify novel early diagnostic markers and therapeutic targets for use in liver cancer diagnosis and treatment (4).

Abnormal tumor-suppressor gene inactivation or oncogene activation are leading causes of liver cancer, and a growing number of studies have indicated that circular RNAs (circRNAs) 
are associated with a variety of cellular activities (5). circRNAs are a large class of endogenous, non-coding RNAs that feature covalently closed continuous loops with highly conserved sequences; they have been revealed to serve an important role in the regulation of gene expression during and after transcription (6). The majority of circRNAs derive from exons, localize in the cytoplasm, and exhibit tissue/developmental stage-specific expression (7). circRNAs can be detected in human blood samples and exhibit higher expression levels than corresponding linear mRNAs (8). As previously reported, circRNAs have been demonstrated to serve a role in the occurrence and progression of a variety of tumor types, including esophageal squamous cell and basal cell carcinoma, as well as colon, ovarian and breast cancers (9-13). These studies clearly demonstrate the potential use of circRNAs as tumor biomarkers (14-16). It has recently been reported that hsa_circ_0001649, hsa_circ_0005075, hsa_ circ_0000130,hsa_circ_0004018 and hsa_circ_0001445 may represent potential biomarkers for hepatocellular carcinoma (HCC), and potentially influence PHC tumor occurrence and metastasis (17-21). circRNAs act as a sponge for microRNAs (miRNAs), regulating downstream mRNA genes through a competing endogenous RNA (ceRNA) network (22). Compared with miRNAs and long non-coding RNAs, circRNAs have not been extensively researched in HCC, with only a few studies focusing on their differential expression in liver cancer tissues. However, to the best of our knowledge, their expression in plasma has not yet been determined, and the use of ceRNA networks and their potential application in clinical diagnosis are yet to be elucidated.

In the present study, comprehensive circRNA expression profiles were analyzed in PHC tissues and paired adjacent normal tissues using circRNA chip screening. Differentially expressed (DE) circRNAs were further validated in PHC tissues and plasma samples using reverse transcription-quantitative (RT-q) PCR. Significant downregulation of hsa_circ_0003056 in PHC tissues and hsa_circ_0067127 in plasma was demonstrated. The ceRNA networks of hsa_circ_0003056 and hsa_circ_0067127 were constructed and analyzed using gene ontology (GO) and Kyoto Encyclopedia of Genes and Genomes (KEGG) pathway analyses.

\section{Materials and methods}

Patients and specimens. A total of 14 PHC tissue specimens and paired adjacent normal tissues, along with fresh plasma samples from 35 PHC patients and 32 healthy donors, were obtained between October 2016 and August 2018 at The Second Hospital of Nanjing (Nanjing, China). Non-tumorous tissues were removed $2.0 \mathrm{~cm}$ from the tumor edge and no obvious malignant cells were identified by a pathologist. Following dissection, all tissues were preserved in RNA storage solution (Shanghai SangonBiotech Co., Ltd.) and stored at $-80^{\circ} \mathrm{C}$ until further use. All tissues were confirmed by pathological examination and none of the patients received neoadjuvant therapy. The baseline characteristics and clinicopathological features of each patient were collected from the hospital digital health care system, and are summarized in Table I. The research procedures of the present study were approved by the Ethics Committee of the Second Hospital of Nanjing, and written informed consent was obtained from all enrolled participants.
circRNA expression profiles. A circRNA chip (Arraystar Human circRNAs Array V2; Zhejiang Kangchen Biotech Co., Ltd.) containing 13,617 probes specific to human circRNA splicing sites was used. A total of three pairs of tumor tissues and paired non-tumorous tissues from patients with PHC were analyzed using the circRNA chips, according to the manufacturer's protocol. The chips were then scanned using the Agilent Scanner G2505C (Agilent Technologies, Inc.), and the raw data were extracted using Agilent Feature Extraction software (version 11.5.1.1; Agilent Technologies, Inc.). Quantile normalization of the raw data and subsequent data processing were performed using the R software limma package 3.40.6 (http://www.bioconductor. org/packages/release/bioc/html/limma.html). Subsequently, low intensity filtering was performed, and circRNAs that appeared in $\geq 3$ out of 6 samples, and had flags in ' $\mathrm{P}$ ' or 'M' ('All Targets Value') were selected for further analyses. circRNAs that exhibited fold changes $\geq 1.5$ and $\mathrm{P} \leq 0.05$ were selected as the significant DE circRNAs. circRNA/miRNA interactions were predicted using Arraystar's home-made miRNA target prediction software, which was derived by modifying an existing software package from TargetScan (23) (http://www.targetscan.org/) and miRanda (24) (http://www. microrna. org/microrna/home.do). The miRNA support vector regression scores were identified and used to construct a 'Top 5' circRNA-miRNA-mRNA network for DE circRNAs.

Total RNA extraction and RT-qPCR. Total RNA was isolated from tumor tissues, adjacent normal tissues and plasma samples using the AllPure Tissue kit, HiPure Blood/Liquid RNA kit (Magen; http://www.magentec.com.cn/) and miRNeasy Serum/Plasma kit (Qiagen, $\mathrm{GmbH}$ ) according to the manufacturers' protocols. The total RNA was quantified using a Colibri spectrometer (Titertek Berthold), and RNA integrity was assessed using electrophoresis on a denaturing agarose gel. Isolated RNA samples were stored at $-80^{\circ} \mathrm{C}$ prior to use. Total RNA from tissue $(1.0 \mu \mathrm{g})$ or plasma $(0.3 \mu \mathrm{g})$ was reverse transcribed into first-strand cDNA using the HiScript Q RT SuperMix for qPCR (+gDNA wiper; Vazyme) according to the manufacturer's protocol. qPCR was performed using the SYBR ${ }^{\circledR}$ Green Master Mix (High ROX Premixed; Vazyme), with the Applied Biosystems ${ }^{\mathrm{TM}}$ QuantStudiot ${ }^{\mathrm{TM}} 3$ Real-Time PCR system (Thermo Fisher Scientific, Inc.). circRNAs were analyzed with $\beta$-actin as the internal standard. The reactions were prepared as follows: $10 \mu \mathrm{l}$ qPCR SYBR ${ }^{\circledR}$ Green Master Mix (High Rox Premixed), $0.4 \mu$ l forward primer $(10 \mu \mathrm{M})$, $0.4 \mu \mathrm{l}$ reverse primer $(10 \mu \mathrm{M}), 8.2 \mu \mathrm{l}$ RNase free water and $1 \mu \mathrm{l}$ cDNA. The thermocycling conditions were as follows: $95^{\circ} \mathrm{C}$ for $5 \mathrm{~min}$, followed by 40 cycles of $95^{\circ} \mathrm{C}$ for $10 \mathrm{sec}$ and $60^{\circ} \mathrm{C}$ for $30 \mathrm{sec}$, and a final step of $95^{\circ} \mathrm{C}$ for $15 \mathrm{sec}, 60^{\circ} \mathrm{C}$ for $60 \mathrm{sec}$ and $95^{\circ} \mathrm{C}$ for $15 \mathrm{sec}$. All primers used in the present study were designed and synthesized by Geneseed Biotech Co., Ltd. and are listed in Table II.

Functional enrichment analyses and ceRNA network construction. GO term enrichment analysis (http://www. geneontology.org) covers three domains: Biological process (BP), cellular component (CC) and molecular function (MF). KEGG pathway enrichment analyses were conducted using the Database for Annotation, Visualization and 
Table I. Baseline characteristics of PHC patients and stratified analyses of hsa_circ_0003056 and has_circ_0067127 expression in PHC plasma.

\begin{tabular}{|c|c|c|c|c|c|c|c|}
\hline \multirow{2}{*}{$\begin{array}{l}\text { Clinicopathological } \\
\text { parameters }\end{array}$} & \multirow[b]{2}{*}{ Chips $(n=3)$} & \multirow{2}{*}{$\begin{array}{c}\text { PHC } \\
\text { tissues }(n=11)\end{array}$} & \multirow{2}{*}{$\begin{array}{c}\text { PHC } \\
\text { plasma }(n=35)\end{array}$} & \multicolumn{2}{|c|}{ Hsa_circ_0003056 } & \multicolumn{2}{|c|}{ Hsa_circ_0067127 } \\
\hline & & & & Mean \pm SD & P-value & Mean \pm SD & P-value \\
\hline \multicolumn{8}{|l|}{ Sex } \\
\hline Male & 3 & 7 & 27 & $2.41 \pm 0.56$ & \multirow[t]{2}{*}{0.66} & $0.31 \pm 0.07$ & \multirow[t]{2}{*}{0.08} \\
\hline Female & 0 & 4 & 8 & $1.89 \pm 1.12$ & & $0.70 \pm 0.25$ & \\
\hline \multicolumn{8}{|l|}{ Age, years } \\
\hline$<60$ & 3 & 11 & 20 & $3.13 \pm 0.80$ & \multirow[t]{2}{*}{0.04} & $0.40 \pm 0.11$ & \multirow[t]{2}{*}{0.56} \\
\hline$\geq 60$ & 0 & 0 & 15 & $1.17 \pm 0.27$ & & $0.30 \pm 0.10$ & \\
\hline \multicolumn{8}{|l|}{$\mathrm{HBsAg}$} \\
\hline Negative & 0 & 1 & 9 & $1.32 \pm 0.44$ & \multirow[t]{2}{*}{0.25} & $0.39 \pm 0.16$ & \multirow[t]{2}{*}{0.82} \\
\hline Positive & 3 & 10 & 26 & $2.63 \pm 0.64$ & & $0.35 \pm 0.09$ & \\
\hline \multicolumn{8}{|l|}{ Cirrhosis } \\
\hline Negative & 1 & 8 & 25 & $1.83 \pm 0.67$ & \multirow[t]{2}{*}{0.56} & $0.51 \pm 0.16$ & \multirow[t]{2}{*}{0.3} \\
\hline Positive & 2 & 3 & 10 & $2.47 \pm 0.64$ & & $0.32 \pm 0.09$ & \\
\hline \multicolumn{8}{|l|}{ AFP, ng/ml } \\
\hline$<200$ & 3 & 6 & 24 & $2.20 \pm 0.58$ & \multirow[t]{2}{*}{0.78} & $0.46 \pm 0.10$ & \multirow[t]{2}{*}{0.05} \\
\hline$\geq 200$ & 0 & 5 & 11 & $2.50 \pm 0.98$ & & $0.16 \pm 0.04$ & \\
\hline \multicolumn{8}{|l|}{ Tumor number } \\
\hline Single & 3 & 8 & 13 & $3.03 \pm 1.16$ & \multirow[t]{2}{*}{0.25} & $0.42 \pm 0.14$ & \multirow[t]{2}{*}{0.64} \\
\hline Multiple & 0 & 3 & 22 & $1.85 \pm 0.39$ & & $0.34 \pm 0.09$ & \\
\hline \multicolumn{8}{|l|}{ Tumor diameter } \\
\hline$<5 \mathrm{~cm}$ & 2 & 6 & 21 & $2.14 \pm 0.62$ & \multirow[t]{2}{*}{0.72} & $0.40 \pm 0.12$ & \multirow[t]{2}{*}{0.61} \\
\hline$\geq 5 \mathrm{~cm}$ & 1 & 5 & 14 & $2.51 \pm 0.84$ & & $0.32 \pm 0.09$ & \\
\hline \multicolumn{8}{|l|}{ TNM stage } \\
\hline I-II & 3 & 11 & 19 & $2.53 \pm 0.83$ & \multirow[t]{2}{*}{0.61} & $0.42 \pm 0.12$ & \multirow[t]{2}{*}{0.38} \\
\hline III-IV & 0 & 0 & 16 & $2.01 \pm 0.47$ & & $0.29 \pm 0.09$ & \\
\hline
\end{tabular}

HBsAg, hepatitis B virus surface antigen; AFP, alpha fetal protein; SD, standard deviation; TNM, stage is a classification of malignant tumors; $\mathrm{T}$, size or direct extent of the primary tumor; $\mathrm{N}$, degree of spread to regional lymph nodes; $\mathrm{M}$, presence of distant metastasis.

Table II. Primer sequences for reverse transcription-quantitative PCR.

\begin{tabular}{|c|c|c|c|c|}
\hline Primer name & Forward (5'-3') & Reverse (5'-3') & Product length & Regulation \\
\hline hsa_circ_0003056 & atttatcacctaaagagccgt & tcaattccttctccaccac & $119 \mathrm{bp}$ & Down \\
\hline hsa_circ_0005090 & gaggagctcaccatctgcta & gagttgcagatcaccttgtcc & $131 \mathrm{bp}$ & Up \\
\hline hsa_circ_0007646 & ggaatgacttctctccaattttca & aagaactgcaagaccgcaga & $152 \mathrm{bp}$ & Up \\
\hline hsa_circ_0064557 & gcccctttcacaggtgatct & ttctgctccaggcgggcaat & $145 \mathrm{bp}$ & Down \\
\hline hsa_circ_0067127 & gtcctccaggatctggctg & hcgcagcttcctcactaacagc & 127 bp & Down \\
\hline
\end{tabular}

Integrated Discovery 6.8 (25), and the P-value (EASE-score, Fisher-P-value or Hypergeometric-P-value) denotes the significance of the pathway when correlated with the conditions. miRNAs that target circRNAs were predicted by surveying for 7-mer or 8-mer complementarity to seed region and the 3' pairing of each miRNA using TargetScan. For humans and mice, two databases were used to predict the target genes of miRNAs: TargetScan 7.1 (http://www.tatgetscan. org/vert_71/) and mirdb V5 (http://mirdb.org/miRDB). The overlapping results of the two databases for humans and mice were accepted. The miRNA-target interactions were then experimentally validated using the database miraTarbase 7.0 (http://mirtarbase.mbc.nctu.edu.tw/php/index.php).

Statistical analysis. Statistical analyses were performed using SPSS version 12.0 (SPSS, Inc.), GraphPad Prism version 5.0 (GraphPad, Inc.) and Cytoscape 2.8.1 (https://cytoscape.org/). Data of the relative expression of circRNAs were analyzed 
using the $2^{-\Delta \Delta \mathrm{Cq}}$ method (26) and presented as the mean \pm standard deviation. Paired or unpaired Student's t-tests were used to compare continuous variables in tissue and plasma samples. An unpaired Student's t-test was used for stratified analyses according to the clinicopathological features of patients with PHC. $\mathrm{P}<0.05$ was considered to indicate a statistically significant result.

\section{Results}

Identification of DE circRNAs in PHC tissues using circRNA chip screening. The expression patterns of circRNAs were determined using circRNA chip screening in three pairs of PHC tissues and adjacent non-tumorous tissues. Following microarray scanning and normalization, significant changes were observed. A total of 155 circRNAs were indicated to be DE in PHC tissues (80 upregulated and 75 downregulated), compared with the paired non-tumorous tissues (Table III). A differential gene expression hierarchical cluster heat map of circRNA expression patterns clearly distinguished PHC tissues from paired adjacent normal tissues (Fig. 1A). In the volcano plot, green and red dots indicate the down- and upregulation of circRNAs expression in PHC tissues, respectively (Fig. 1B).

Confirmation of DE circRNAs in PHC tissues using RT-qPCR. To confirm the differential expression of circRNAs (indicated by circRNA microarray screening), RT-qPCR was performed to assess the expression levels of five candidate DE circRNAs in PHC tissues, compared with adjacent normal tissues, derived from an additional 11 patients with PHC. The five circRNAs (two upregulated and three downregulated) were selected according to the following criteria: i) Fold-change $>1.5$; ii) $\mathrm{P}<0.05$; iii) exonic-related circRNAs; iv) raw intensity $\geq 100$; v) included in circBase; and vi) exhibiting a gene symbol or miRNA binding sites that were considered to be significantly associated with tumor occurrence. Only circRNAs that exhibited a $\geq 1.5$-fold expression level difference were considered to display statistically significant changes $(\mathrm{P}<0.05)$. Moreover, complete and accurate sequence information, and other information regarding the selected exonic-related circRNAs, were retrieved from circBase. A group raw intensity of $\geq 100$ ensured the reliability of the detected fluorescence values of circRNAs determined using the microassay. Finally, circRNAs may influence PHC development and progression through circRNA-miRNA-mRNA networks. Thus, only circRNAs whose target genes or miRNA binding sites had a predetermined association with cancer progression were preferentially selected.

As exhibited in Fig. 2A, only hsa_circ_0003056 expression was consistently and significantly lower in PHC tissues when compared with paired adjacent non-tumorous tissues. The other three circRNAs exhibited a fold-change in expression level between 1.5 and 2 , which was not significantly different from the adjacent tissues.

Expression levels and association with clinicopathological parameters of hsa_circ_0003056 and hsa_circ_0067127 in PHC patient plasma. Hsa_circ_0003056 expression levels were decreased in PHC tissues compared with paired, adjacent non-tumor tissues. Hsa_circ_0067127 also exhibited decreased expression levels in PHC tissues, but the difference was not significant compared with the adjacent non-tumorous tissues, which may be due to the small sample size. To determine whether changes in expression level were also reflected in patients' plasma, fresh plasma samples were collected from an additional 35 patients with PHC, and 32 healthy donors. Subsequently, the expression levels of hsa_circ_0003056 and hsa_circ_0067127 were detected using RT-qPCR. As indicated in Fig. 2B, no significant downregulation or upregulation of hsa_circ_0003056 was identified in the patients' plasma, when compared with healthy donor plasma. However, hsa_circ_0067127 was significantly downregulated in the PHC plasma. Stratified analyses of hsa_circ_0003056 and hsa_circ_0067127 levels in the plasma samples of patients with PHC was performed, and is summarized in Table I. Significant downregulation of hsa_circ_0003056 was observed in patients who were $>60$ years old compared those $<60$ years old. hsa_circ_0067127 levels were also significantly higher in patients with an AFP level $<200 \mathrm{ng} / \mathrm{ml}$. No association was demonstrated between other clinical and laboratory characteristics (including sex and HBsAg level) and hsa_circ_0003056.

Follow-up was carried out for the 35 patients with PHC and the median overall survival (OS) time was 69 months. Survival data were analyzed using the log-rank test and survival curves were generated using the Kaplan-Meier method. Regarding hsa_circ_0003056 expression, the corresponding 35 patients with PHC were separated into an hsa_circ_0003056 low-expression group (<mean value in healthy donors' plasma; $\mathrm{n}=15$; OS=69) and hsa_circ_0003056 high-expression group (>mean value in healthy donors' plasma; $n=20$; OS=72). The difference between OS times observed between groups was not statistically significant (data not shown). In terms of hsa_circ_0067127 expression, only 3 out of 35 patients with PHC displayed higher expression in their plasma than the mean value in the healthy donors. As a result, the survival curves could not be analyzed.

Construction of ceRNA networks of hsa_circ_0003056 and hsa_circ_0067127, and KEGG/GO enrichment analyses. The target miRNAs of hsa_circ_0003056 and hsa_circ_0067127 were identified and ranked based on their mirSVR scores and TargetScan. This indicated that hsa_circ_0003056 and hsa_circ_0067127 serve as miRNA sponges, regulating the ceRNA network. The five top miRNAs were selected to establish circRNA-miRNA-mRNA networks: hsa-miR-211-5p, hsa-miR-204-5p, hsa-miR-9-3p, hsa-miR-2113 and hsa-miR-499a-5p targeting to hsa_circ_0003056 (Fig. 3A), and hsa-miR-141-5p, hsa-miR-486-5p, hsa-miR-186-3p, hsa-miR-647 and hsa-miR-212-5p targeting to hsa_circ_0067127 (Fig. 3B). Regarding hsa_circ_0003056, a Venn diagram (Fig. 4A) indicated that overlapping results common to the two databases were accepted. TargetScan 7.1 and mirdbV5 are used to forecast the target genes of miRNAs. The bar plot presents the enrichment scores [- $\log _{10}$ (P-value)] of the top 10 significant enrichment pathways. KEGG pathway analysis demonstrated that DE genes were significantly enriched in pathways regulating the pluripotency of stem cells (Fig. 4B). The bar plot presents the enrichment score [-log10 (P-value)] values of the top 10 significant $\mathrm{GO}$ enrichments. The hsa_circ_0003056-miRNAs-targets network exhibited a strong 
Table III. Differentially expressed circRNAs.

\begin{tabular}{|c|c|c|c|c|c|c|c|}
\hline $\begin{array}{l}\text { CircRNA ID } \\
\text { (circBase) }\end{array}$ & Chromosome & Strand & circRNA type & Gene symbol & P-value & $\begin{array}{c}\text { Fold } \\
\text { change }(\mathrm{FC})\end{array}$ & Regulation \\
\hline hsa_circ_0000745 & $\operatorname{chr} 17$ & + & Exonic & SPECC1 & 0.0212245 & 1.9054513 & Up \\
\hline$\# \mathrm{~N} / \mathrm{A}$ & $\operatorname{chr} 1$ & - & Exonic & PIK3C2B & 0.025524 & 3.1458593 & Up \\
\hline hsa_circ_0092125 & $\operatorname{chrX}$ & - & Exonic & G6PD & 0.0155316 & 2.3746559 & $\mathrm{Up}$ \\
\hline hsa_circ_0068293 & $\operatorname{chr} 3$ & + & Exonic & AP2M1 & 0.0373273 & 1.7639796 & Up \\
\hline hsa_circ_0007693 & chr1 & - & Exonic & ERI3 & 0.0404268 & 1.6569751 & Up \\
\hline hsa_circ_0025768 & $\operatorname{chr} 12$ & - & Exonic & TMTC1 & 0.0265629 & 1.5242278 & Up \\
\hline hsa_circ_0005075 & $\operatorname{chr} 1$ & - & Exonic & EIF4G3 & 0.0360363 & 2.8689281 & Up \\
\hline hsa_circ_0073030 & $\operatorname{chr} 5$ & - & Exonic & FAM169A & 0.0044122 & 2.731725 & Up \\
\hline hsa_circ_0008106 & $\operatorname{chr} 3$ & + & Exonic & LRCH3 & 0.0472836 & 2.2037281 & $\mathrm{Up}$ \\
\hline hsa_circ_0001231 & $\operatorname{chr} 22$ & - & Exonic & DMC1 & 0.0239959 & 1.9820607 & Up \\
\hline hsa_circ_0007646 & chr4 & + & Exonic & DCUN1D4 & 0.0433039 & 1.9509878 & Up \\
\hline hsa_circ_0008439 & $\operatorname{chr} 3$ & + & Exonic & LRCH3 & 0.0402462 & 1.8599736 & Up \\
\hline hsa_circ_0028711 & $\operatorname{chr} 12$ & - & Exonic & RAB35 & 0.0040302 & 1.6338613 & Up \\
\hline hsa_circ_0000760 & $\operatorname{chr} 17$ & + & Overlapping & MLLT6 & 0.0058239 & 1.6282967 & Up \\
\hline hsa_circ_0005873 & chr3 & + & Exonic & LRCH3 & 0.0368806 & 2.4621779 & $\mathrm{Up}$ \\
\hline hsa_circ_0002563 & $\operatorname{chr} 1$ & + & Exonic & KIF2C & 0.0035669 & 2.5852184 & Up \\
\hline hsa_circ_0076522 & chr6 & + & Exonic & ABCC10 & 0.0204543 & 2.2221132 & Up \\
\hline hsa_circ_0008719 & chr19 & - & Exonic & AKT2 & 0.0326238 & 1.8104931 & Up \\
\hline hsa_circ_0011480 & $\operatorname{chr} 1$ & - & Exonic & PHC2 & 0.0495597 & 1.5408582 & Up \\
\hline hsa_circ_0011477 & chr1 & - & Exonic & PHC2 & 0.0465285 & 1.5066423 & Up \\
\hline hsa_circ_0081626 & $\operatorname{chr} 7$ & + & Exonic & CUX1 & 0.0458484 & 1.5696195 & Up \\
\hline hsa_circ_0002994 & $\operatorname{chr} 17$ & - & Exonic & ACLY & 0.0228593 & 2.0403136 & Up \\
\hline hsa_circ_0043101 & $\operatorname{chr} 17$ & - & Exonic & NLE1 & 0.015046 & 1.539322 & Up \\
\hline hsa_circ_0000937 & $\operatorname{chr} 19$ & + & Exonic & BCKDHA & 0.024951 & 1.8707604 & Up \\
\hline hsa_circ_0008958 & $\operatorname{chr} 1$ & + & Overlapping & CIART & 0.0002 & 1.5087793 & Up \\
\hline hsa_circ_0003209 & $\operatorname{chr} 20$ & + & Exonic & TPX2 & 0.0411834 & 1.5429038 & $\mathrm{Up}$ \\
\hline hsa_circ_0043497 & $\operatorname{chr} 17$ & - & Exonic & MED24 & 0.0446081 & 2.4071688 & Up \\
\hline$\# \mathrm{~N} / \mathrm{A}$ & chr7 & + & Exonic & AP1S1 & 0.005098 & 2.4339844 & Up \\
\hline hsa_circ_0007328 & chr19 & + & Exonic & DOT1L & 0.0098403 & 1.7198141 & Up \\
\hline hsa_circ_0087080 & chr9 & - & Exonic & FBXO10 & 0.0361757 & 1.6176128 & $\mathrm{Up}$ \\
\hline hsa_circ_0028990 & $\operatorname{chr} 12$ & - & Exonic & KDM2B & 0.0075388 & 1.6618626 & Up \\
\hline hsa_circ_0001591 & chr6 & + & Overlapping & HIST1H4I & 0.0229166 & 1.9052747 & Up \\
\hline hsa_circ_0060456 & $\operatorname{chr} 20$ & + & Exonic & MYBL2 & 0.0131702 & 2.3768838 & Up \\
\hline hsa_circ_0017289 & $\operatorname{chr} 1$ & - & Exonic & SMYD3 & 0.0356072 & 1.9878667 & Up \\
\hline hsa_circ_0037409 & chr16 & + & Exonic & TSC2 & 0.0212241 & 2.4079542 & Up \\
\hline hsa_circ_0082614 & $\operatorname{chr} 7$ & - & Exonic & KIAA1549 & 0.0017741 & 1.5392865 & Up \\
\hline hsa_circ_0004656 & chr16 & + & Exonic & SLC7A6 & 0.0019672 & 1.977973 & Up \\
\hline hsa_circ_0071653 & $\operatorname{chr} 5$ & + & Exonic & CEP72 & 0.0224188 & 1.9589469 & $\mathrm{Up}$ \\
\hline hsa_circ_0003048 & $\operatorname{chr} 16$ & - & Exonic & VAC14 & 0.0495136 & 1.7512677 & Up \\
\hline hsa_circ_0006916 & $\operatorname{chr} 5$ & - & Exonic & HOMER1 & 0.0127235 & 2.0032953 & Up \\
\hline hsa_circ_0050898 & chr19 & + & Exonic & ACTN4 & 0.0383419 & 2.5628111 & Up \\
\hline hsa_circ_0069370 & $\operatorname{chr} 4$ & - & Exonic & SEL1L3 & 0.0033967 & 1.5398487 & Up \\
\hline \#N/A & chr19 & + & Intronic & DHX34 & 0.0467045 & 1.766391 & Up \\
\hline hsa_circ_0089090 & $\operatorname{chr} 9$ & + & Exonic & NCS1 & 0.0028527 & 1.522742 & Up \\
\hline hsa_circ_0092297 & $\operatorname{chr} 2$ & - & Intronic & SMPD4 & 0.0208144 & 1.7537756 & Up \\
\hline hsa_circ_0051718 & chr19 & - & Exonic & LIG1 & 0.006625 & 1.8456723 & Up \\
\hline hsa_circ_0011279 & $\operatorname{chr} 1$ & + & Exonic & SERINC2 & 0.0451375 & 1.5002793 & Up \\
\hline hsa_circ_0088072 & $\operatorname{chr} 9$ & - & Exonic & PTBP3 & 0.0046249 & 1.603221 & Up \\
\hline hsa_circ_0017287 & chr1 & - & Exonic & SMYD3 & 0.0350386 & 1.7546953 & Up \\
\hline hsa_circ_0005090 & $\operatorname{chr} 1$ & - & Exonic & SMYD3 & 0.0144482 & 1.6936278 & Up \\
\hline hsa_circ_0002688 & $\operatorname{chr} 4$ & + & Exonic & WHSC1 & 0.0121876 & 3.363273 & $\mathrm{Up}$ \\
\hline
\end{tabular}


Table III. Continued.

\begin{tabular}{|c|c|c|c|c|c|c|c|}
\hline $\begin{array}{l}\text { CircRNA ID } \\
\text { (circBase) }\end{array}$ & Chromosome & Strand & circRNA type & Gene symbol & P-value & $\begin{array}{c}\text { Fold } \\
\text { change (FC) }\end{array}$ & Regulation \\
\hline \#N/A & $\operatorname{chr} 5$ & - & Exonic & LPCAT1 & 0.0447121 & 1.8767479 & Up \\
\hline hsa_circ_0006177 & $\operatorname{chr} 2$ & + & Intronic & AGAP1 & 0.0084361 & 1.5176364 & Up \\
\hline hsa_circ_0002245 & chr6 & + & Exonic & CAP2 & 0.0433245 & 1.6219694 & Up \\
\hline hsa_circ_0014754 & chr1 & - & Exonic & IQGAP3 & 0.0036918 & 2.5346514 & Up \\
\hline hsa_circ_0092324 & $\operatorname{chr} 17$ & - & Intronic & DNAJC7 & 0.0216354 & 2.380195 & $\mathrm{Up}$ \\
\hline$\# \mathrm{~N} / \mathrm{A}$ & $\operatorname{chr} 5$ & - & Exonic & $\mathrm{CDC} 25 \mathrm{C}$ & 0.0454826 & 1.8293722 & Up \\
\hline hsa_circ_0041008 & $\operatorname{chr} 16$ & - & Exonic & FANCA & 0.0127351 & 1.8125889 & $\mathrm{Up}$ \\
\hline hsa_circ_0055855 & chr2 & - & Exonic & AFF3 & 0.0003327 & 2.8560336 & Up \\
\hline hsa_circ_0059760 & $\operatorname{chr} 20$ & + & Exonic & TM9SF4 & 0.0219282 & 2.247014 & Up \\
\hline$\# \mathrm{~N} / \mathrm{A}$ & $\operatorname{chr} 21$ & - & Exonic & HSF2BP & 0.0387871 & 1.6720688 & $\mathrm{Up}$ \\
\hline hsa_circ_0043947 & $\operatorname{chr} 17$ & - & Exonic & BRCA1 & 0.0408289 & 1.5983177 & $\mathrm{Up}$ \\
\hline$\# \mathrm{~N} / \mathrm{A}$ & $\operatorname{chr} 4$ & + & Exonic & TACC3 & 0.0080579 & 3.2048942 & Up \\
\hline hsa_circ_0001728 & $\operatorname{chr} 7$ & - & Intronic & MCM7 & 0.0318177 & 2.2162243 & $\mathrm{Up}$ \\
\hline hsa_circ_0092370 & $\operatorname{chr} 1$ & - & Intronic & DNAJC11 & 0.0188763 & 1.8250598 & Up \\
\hline$\# \mathrm{~N} / \mathrm{A}$ & chr19 & - & Exonic & CADM4 & 0.0287784 & 1.8768288 & Up \\
\hline$\# \mathrm{~N} / \mathrm{A}$ & chr4 & - & Exonic & SEL1L3 & 0.0412118 & 1.711422 & Up \\
\hline hsa_circ_0082688 & chr7 & - & Exonic & PARP12 & 0.0259324 & 1.5632151 & $\mathrm{Up}$ \\
\hline$\# \mathrm{~N} / \mathrm{A}$ & $\operatorname{chr} 12$ & + & Exonic & $\mathrm{P} 2 \mathrm{RX} 4$ & 0.0057199 & 1.6028692 & Up \\
\hline hsa_circ_0006893 & chr3 & - & Exonic & PHC3 & 0.0133705 & 1.5220367 & Up \\
\hline hsa_circ_0082304 & chr7 & + & Exonic & NRF1 & 0.0164259 & 1.8654058 & $\mathrm{Up}$ \\
\hline hsa_circ_0001196 & $\operatorname{chr} 21$ & - & Exonic & WDR4 & 0.0471616 & 2.1165344 & Up \\
\hline hsa_circ_0068894 & chr4 & + & Exonic & WHSC1 & 0.0476107 & 3.2029375 & Up \\
\hline hsa_circ_0087047 & $\operatorname{chr} 9$ & + & Exonic & $\mathrm{ZCCHC7}$ & 0.0103933 & 1.7475255 & $\mathrm{Up}$ \\
\hline hsa_circ_0002071 & $\operatorname{chr} 12$ & - & Exonic & GOLGA3 & 0.0050376 & 2.0771037 & Up \\
\hline hsa_circ_0012166 & chr1 & + & Exonic & KIF2C & 0.0324916 & 1.6186238 & Up \\
\hline hsa_circ_0008777 & $\operatorname{chr} 20$ & - & Exonic & AURKA & 0.0376056 & 1.9286644 & Up \\
\hline hsa_circ_0005219 & $\operatorname{chr} 20$ & + & Exonic & STK35 & 0.0086497 & 2.0307961 & Up \\
\hline hsa_circ_0012151 & chr1 & - & Exonic & ERI3 & 0.0237599 & 1.7283688 & Up \\
\hline hsa_circ_0037526 & $\operatorname{chr} 16$ & + & Exonic & $\mathrm{CCNF}$ & 0.0156714 & 2.1195943 & $\mathrm{Up}$ \\
\hline hsa_circ_0002198 & chr6 & + & Exonic & PDE7B & 0.0301888 & 2.2417711 & Down \\
\hline hsa_circ_0004712 & chr6 & + & Exonic & PDE7B & 0.0455082 & 2.5301703 & Down \\
\hline hsa_circ_0039783 & chr16 & + & Exonic & CBFB & 0.0005419 & 2.2404601 & Down \\
\hline$\# \mathrm{~N} / \mathrm{A}$ & $\operatorname{chr} 3$ & - & Intronic & MAGI1 & 0.0456285 & 2.1403102 & Down \\
\hline hsa_circ_0008945 & chr14 & - & Exonic & NIN & 0.017864 & 1.7247068 & Down \\
\hline$\# \mathrm{~N} / \mathrm{A}$ & chr10 & - & Exonic & ANK3 & 0.0335636 & 2.7176467 & Down \\
\hline hsa_circ_0003661 & $\operatorname{chr} 11$ & + & Exonic & ANKRD42 & 0.0446637 & 1.7336621 & Down \\
\hline hsa_circ_0030051 & $\operatorname{chr} 13$ & - & Exonic & ELF1 & 0.03574 & 1.731437 & Down \\
\hline hsa_circ_0000720 & chr16 & + & Exonic & PLCG2 & 0.0304322 & 1.8390616 & Down \\
\hline hsa_circ_0089372 & chr9 & + & Exonic & ADAMTS13 & 0.0252263 & 2.8096697 & Down \\
\hline hsa_circ_0002089 & $\operatorname{chr} 11$ & + & Exonic & ARHGEF12 & 0.0063054 & 2.7586096 & Down \\
\hline hsa_circ_0078223 & chr6 & - & Exonic & LATS1 & 0.0447052 & 1.6186692 & Down \\
\hline$\# \mathrm{~N} / \mathrm{A}$ & $\operatorname{chr} 5$ & - & Exonic & MYO10 & 0.0190623 & 2.9255358 & Down \\
\hline hsa_circ_0059071 & $\operatorname{chr} 2$ & + & Exonic & FARP2 & 0.0414464 & 1.5860201 & Down \\
\hline hsa_circ_0005801 & $\operatorname{chr} 10$ & - & Exonic & TM9SF3 & 0.0396484 & 1.6371578 & Down \\
\hline hsa_circ_0003357 & $\operatorname{chr} 10$ & + & Exonic & ADD3 & 0.0499515 & 1.9810488 & Down \\
\hline hsa_circ_0069323 & chr4 & - & Exonic & GPR125 & 0.0114958 & 1.9450245 & Down \\
\hline hsa_circ_0004219 & chr8 & + & Exonic & DOCK5 & 0.009565 & 3.2333123 & Down \\
\hline hsa_circ_0002955 & $\operatorname{chr} 11$ & + & Exonic & ARHGEF12 & 0.0472601 & 3.0446992 & Down \\
\hline hsa_circ_0017248 & $\operatorname{chr} 1$ & - & Exonic & AKT3 & 0.0481372 & 1.6202917 & Down \\
\hline hsa_circ_0042339 & $\operatorname{chr} 17$ & - & Exonic & SHMT1 & 0.0289513 & 1.6262584 & Down \\
\hline$\# \mathrm{~N} / \mathrm{A}$ & $\operatorname{chr} 2$ & - & Exonic & SMC6 & 0.0354823 & 1.6429599 & Down \\
\hline
\end{tabular}


Table III. Continued.

\begin{tabular}{|c|c|c|c|c|c|c|c|}
\hline $\begin{array}{l}\text { CircRNA ID } \\
\text { (circBase) }\end{array}$ & Chromosome & Strand & circRNA type & Gene symbol & P-value & $\begin{array}{c}\text { Fold } \\
\text { change }(\mathrm{FC})\end{array}$ & Regulation \\
\hline hsa_circ_0003056 & $\operatorname{chr} 22$ & - & Exonic & PITPNB & 0.0021306 & 2.1416189 & Down \\
\hline hsa_circ_0001041 & $\operatorname{chr} 2$ & - & Exonic & EIF2AK3 & 0.0281227 & 1.7488955 & Down \\
\hline hsa_circ_0000550 & $\operatorname{chr} 14$ & + & Antisense & SLC10A1 & 0.0143731 & 2.6344379 & Down \\
\hline hsa_circ_0003640 & $\operatorname{chr} 4$ & + & Exonic & METAP1 & 0.002348 & 1.8630256 & Down \\
\hline hsa_circ_0078328 & chr6 & - & Exonic & SYNE1 & 0.0220923 & 1.7778472 & Down \\
\hline hsa_circ_0036610 & $\operatorname{chr} 15$ & + & Exonic & PDE8A & 0.0491465 & 1.8214826 & Down \\
\hline hsa_circ_0005045 & chr2 & - & Exonic & RTN4 & 0.0407689 & 2.1566167 & Down \\
\hline hsa_circ_0006663 & $\operatorname{chr} 16$ & - & Exonic & LUC7L & 0.0277407 & 1.5172731 & Down \\
\hline hsa_circ_0073649 & $\operatorname{chr} 5$ & - & Exonic & DTWD2 & 0.0126395 & 1.6366199 & Down \\
\hline \#N/A & $\operatorname{chr} 13$ & + & Exonic & EEF1DP3 & 0.0365432 & 1.8802713 & Down \\
\hline hsa_circ_0067127 & $\operatorname{chr} 3$ & - & Exonic & ALDH1L1 & 0.0187756 & 4.2915721 & Down \\
\hline hsa_circ_0038409 & $\operatorname{chr} 16$ & - & Exonic & LOC100271836 & 0.0381125 & 1.731821 & Down \\
\hline hsa_circ_0071311 & $\operatorname{chr} 4$ & + & Exonic & KIAA0922 & 0.007598 & 2.0057656 & Down \\
\hline hsa_circ_0002771 & $\operatorname{chr} 16$ & - & Exonic & PARN & 0.0286315 & 1.7820343 & Down \\
\hline hsa_circ_0005471 & $\operatorname{chr} 17$ & - & Exonic & NCOR1 & 0.0039017 & 1.8690309 & Down \\
\hline hsa_circ_0069244 & $\operatorname{chr} 4$ & - & Exonic & LDB2 & 0.0246507 & 2.4543633 & Down \\
\hline hsa_circ_0015164 & $\operatorname{chr} 1$ & - & Exonic & SLC19A2 & 0.0118506 & 1.9309544 & Down \\
\hline hsa_circ_0006539 & $\operatorname{chr} 8$ & + & Exonic & RBPMS & 0.0471379 & 2.564619 & Down \\
\hline \#N/A & $\operatorname{chr} 4$ & - & Intronic & GPR125 & 0.0314876 & 1.6460992 & Down \\
\hline hsa_circ_0053070 & $\operatorname{chr} 2$ & + & Exonic & HADHB & 0.0223274 & 1.8756974 & Down \\
\hline hsa_circ_0023730 & $\operatorname{chr} 11$ & - & Exonic & INTS4 & 0.0474224 & 1.644243 & Down \\
\hline hsa_circ_0038644 & $\operatorname{chr} 16$ & + & Exonic & PRKCB & 0.0050378 & 1.5424169 & Down \\
\hline hsa_circ_0064557 & $\operatorname{chr} 3$ & - & Exonic & SATB 1 & 0.0344432 & 1.6859964 & Down \\
\hline \#N/A & $\operatorname{chr} 1$ & - & Exonic & PDE4DIP & 0.0220458 & 1.6090404 & Down \\
\hline hsa_circ_0069681 & $\operatorname{chr} 4$ & - & Exonic & FRYL & 0.0374764 & 1.6037813 & Down \\
\hline hsa_circ_0006891 & $\operatorname{chr} 11$ & + & Exonic & PPFIBP2 & 0.0257878 & 1.6666432 & Down \\
\hline hsa_circ_0021827 & $\operatorname{chr} 11$ & - & Exonic & PHF21A & 0.0249203 & 1.589884 & Down \\
\hline hsa_circ_0001122 & $\operatorname{chr} 2$ & + & Exonic & FARP2 & 0.0045619 & 1.5731657 & Down \\
\hline hsa_circ_0013607 & $\operatorname{chr} 1$ & - & Exonic & RSBN1 & 0.0376341 & 2.0202019 & Down \\
\hline hsa_circ_0005967 & $\operatorname{chr} 10$ & - & Exonic & KCNMA1 & 0.0160756 & 1.6827265 & Down \\
\hline hsa_circ_0000446 & $\operatorname{chr} 12$ & - & Exonic & TAOK3 & 0.0325774 & 1.6013056 & Down \\
\hline hsa_circ_0070857 & $\operatorname{chr} 4$ & + & Exonic & KIAA1109 & 0.0418502 & 1.8511599 & Down \\
\hline hsa_circ_0007590 & $\operatorname{chr} 13$ & - & Exonic & LATS2 & 0.007774 & 1.7207037 & Down \\
\hline hsa_circ_0006629 & $\operatorname{chr} 11$ & - & Exonic & PICALM & 0.0191864 & 1.750488 & Down \\
\hline hsa_circ_0001640 & chr6 & - & Exonic & EPB41L2 & 0.0482272 & 2.8931722 & Down \\
\hline hsa_circ_0066378 & $\operatorname{chr} 3$ & + & Exonic & ABHD6 & 0.0429032 & 2.3329893 & Down \\
\hline hsa_circ_0025711 & $\operatorname{chr} 12$ & - & Exonic & TM7SF3 & 0.0253257 & 2.0133054 & Down \\
\hline hsa_circ_0023865 & $\operatorname{chr} 11$ & - & Exonic & CCDC90B & 0.0386574 & 1.6774031 & Down \\
\hline hsa_circ_0000077 & $\operatorname{chr} 1$ & - & Overlapping & TM2D1 & 0.0236483 & 1.5061897 & Down \\
\hline hsa_circ_0005527 & $\operatorname{chr} 11$ & - & Exonic & FCHSD2 & 0.02705 & 2.2952095 & Down \\
\hline hsa_circ_0030042 & $\operatorname{chr} 13$ & - & Exonic & FOXO1 & 0.0174801 & 2.1639648 & Down \\
\hline hsa_circ_0007201 & $\operatorname{chr} 15$ & + & Exonic & IQGAP1 & 0.0248831 & 1.7816364 & Down \\
\hline \#N/A & $\operatorname{chr} 11$ & + & Exonic & DLAT & 0.0309512 & 1.5343122 & Down \\
\hline \#N/A & $\operatorname{chr} 4$ & - & Exonic & NR3C2 & 0.0476049 & 1.5305334 & Down \\
\hline hsa_circ_0084137 & chr8 & - & Exonic & RNF170 & 0.0164973 & 2.1286009 & Down \\
\hline hsa_circ_0013162 & $\operatorname{chr} 1$ & - & Exonic & EVI5 & 0.0337218 & 2.6161063 & Down \\
\hline hsa_circ_0037969 & chr16 & - & Exonic & PARN & 0.0354487 & 1.5344047 & Down \\
\hline hsa_circ_0077765 & chr6 & + & Exonic & RNF217 & 0.0169759 & 1.5605383 & Down \\
\hline$\# \mathrm{~N} / \mathrm{A}$ & $\operatorname{chr} 11$ & + & Exonic & TEAD1 & 0.0014024 & 1.81072 & Down \\
\hline hsa_circ_0035376 & $\operatorname{chr} 15$ & + & Exonic & PIGB & 0.0174 & 1.5311146 & Down \\
\hline hsa_circ_0000389 & $\operatorname{chr} 12$ & + & Over-lapping & FGD4 & 0.0319098 & 1.5397315 & Down \\
\hline hsa_circ_0030281 & $\operatorname{chr} 13$ & - & Exonic & DLEU2 & 0.0245441 & 2.2986061 & Down \\
\hline$\# \mathrm{~N} / \mathrm{A}$ & $\operatorname{chr} 16$ & + & Intronic & MT2A & 0.0025438 & 2.8617763 & Down \\
\hline
\end{tabular}


A

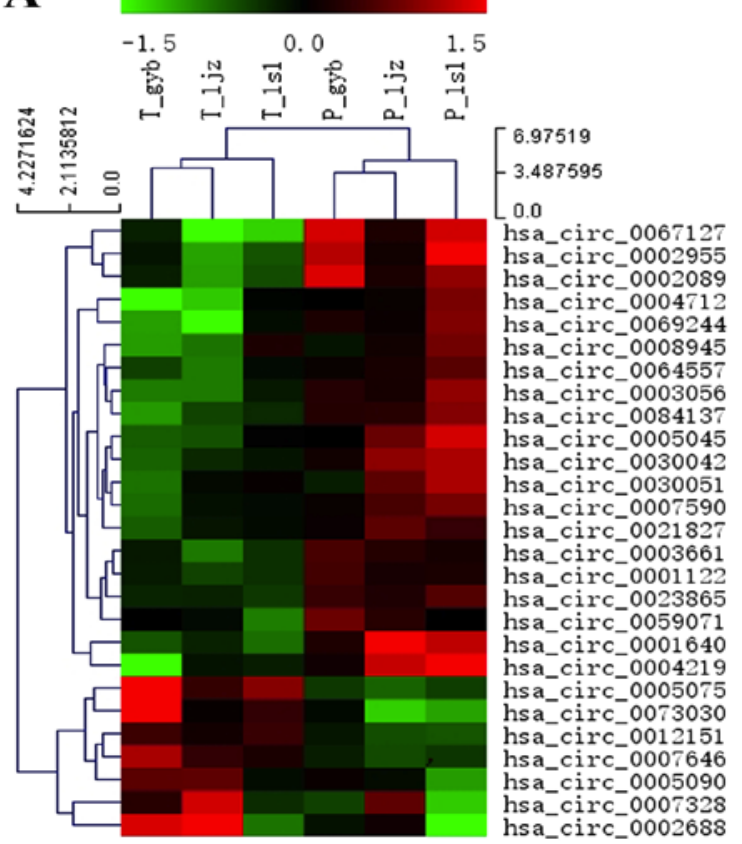

B

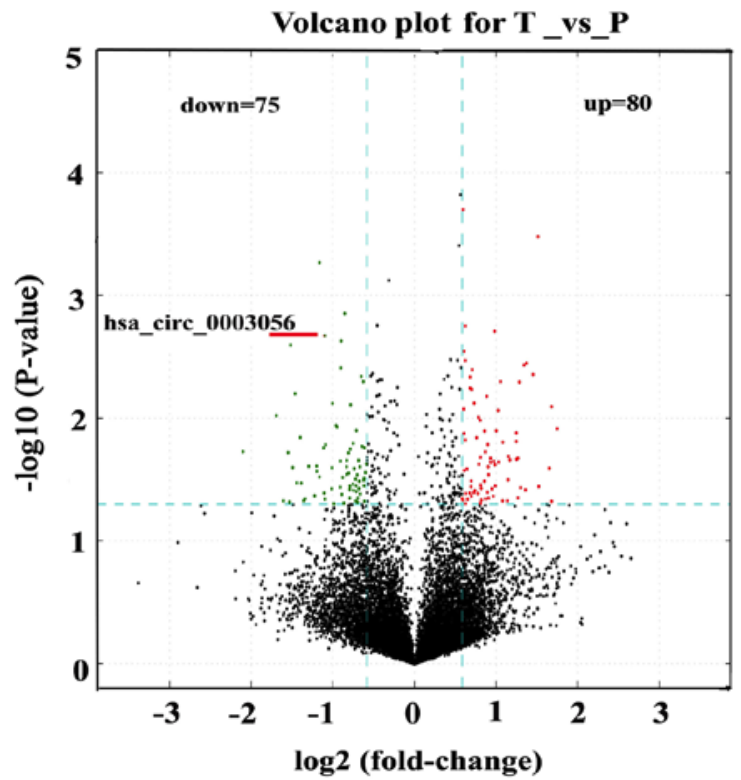

Figure 1. Differential expression of circRNAs in PHC tissues detected by circRNA chip screening. (A) Hierarchical cluster heat map of the top 27 most upregulated and downregulated circRNAs in PHC tissues, compared with matched adjacent non-tumorous tissues, analyzed using circRNAs Arraystar Chip in six samples. (B) Volcano plot of the differential expression of circRNAs between PHC tissues and paired paracancerous tissues: The vertical blue line corresponds to up- and downregulation (fold change $>1.5$ ). The green dots and red dots indicate down- and upregulated circRNAs in PHC tissues, respectively. Horizontal blue line indicates $\mathrm{P}<0.05$. circRNA, circular RNA; PHC, primary hepatic carcinoma; $\mathrm{T}$, tumor tissue; $\mathrm{P}$, paired paracancerous tissues.
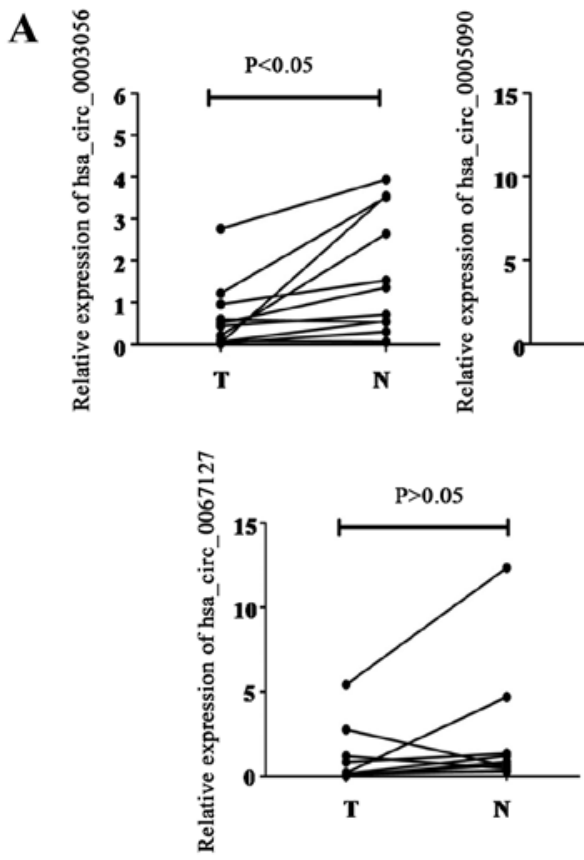
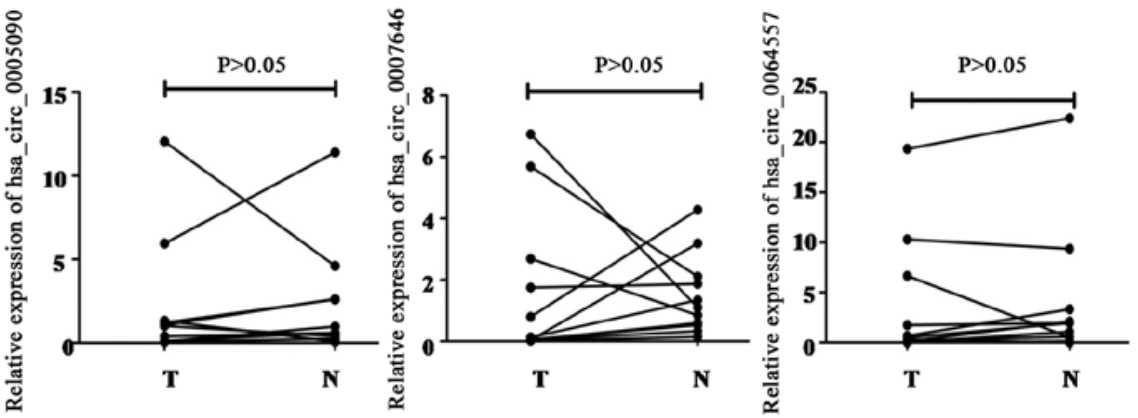

B
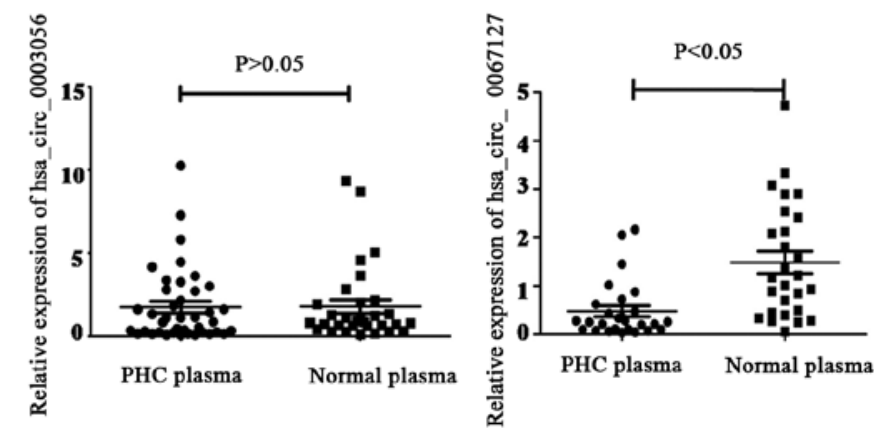

Figure 2. Confirmation of DE circRNAs in PHC tissues and plasma by RT-qPCR. (A) Expression levels of hsa_circ_0003056, hsa_circ_0005090, hsa_ circ_0007646, hsa_circ_0064557 and hsa_circ_0067127 were assessed in 11 PHC patients, with comparisons between PHC tissues and adjacent non-tumor tissues conducted using RT-qPCR; (B) Scatter plots display the relative expression of hsa_circ_0003056 and hsa_circ_0067127 in 35 PHC and 32 healthy plasma samples. DE, differentially expressed; circRNA, circular RNA; PHC, primary hepatic carcinoma; RT-q, reverse transcription-quantitative; T, tumor tissue; $\mathrm{N}$, non-tumorous tissues.

association with 'regulation of kinase activity', 'intracellular-' and 'transmembrane-ephrin receptor' activity in $\mathrm{BP}, \mathrm{CC}$ and MF, respectively (Fig. 4C). The overlapping results between the two databases were also accepted for hsa_circ_0067127
(Fig. 4D). DE genes were significantly enriched in the pathways associated with 'ubiquitin-mediated proteolysis' and 'prostate cancer' (Fig. 4E). The top 10 significant GO enrichments are presented in Fig. 4F. The hsa_circ_0067127-miRNAs-targets 
A hsa-miR-211-5p/hsa-miR-204-5p/hsa-miR-9-3p/hsa-miR-2113/hsa-miR-499a-5p_VS_hsa_circ_0003056

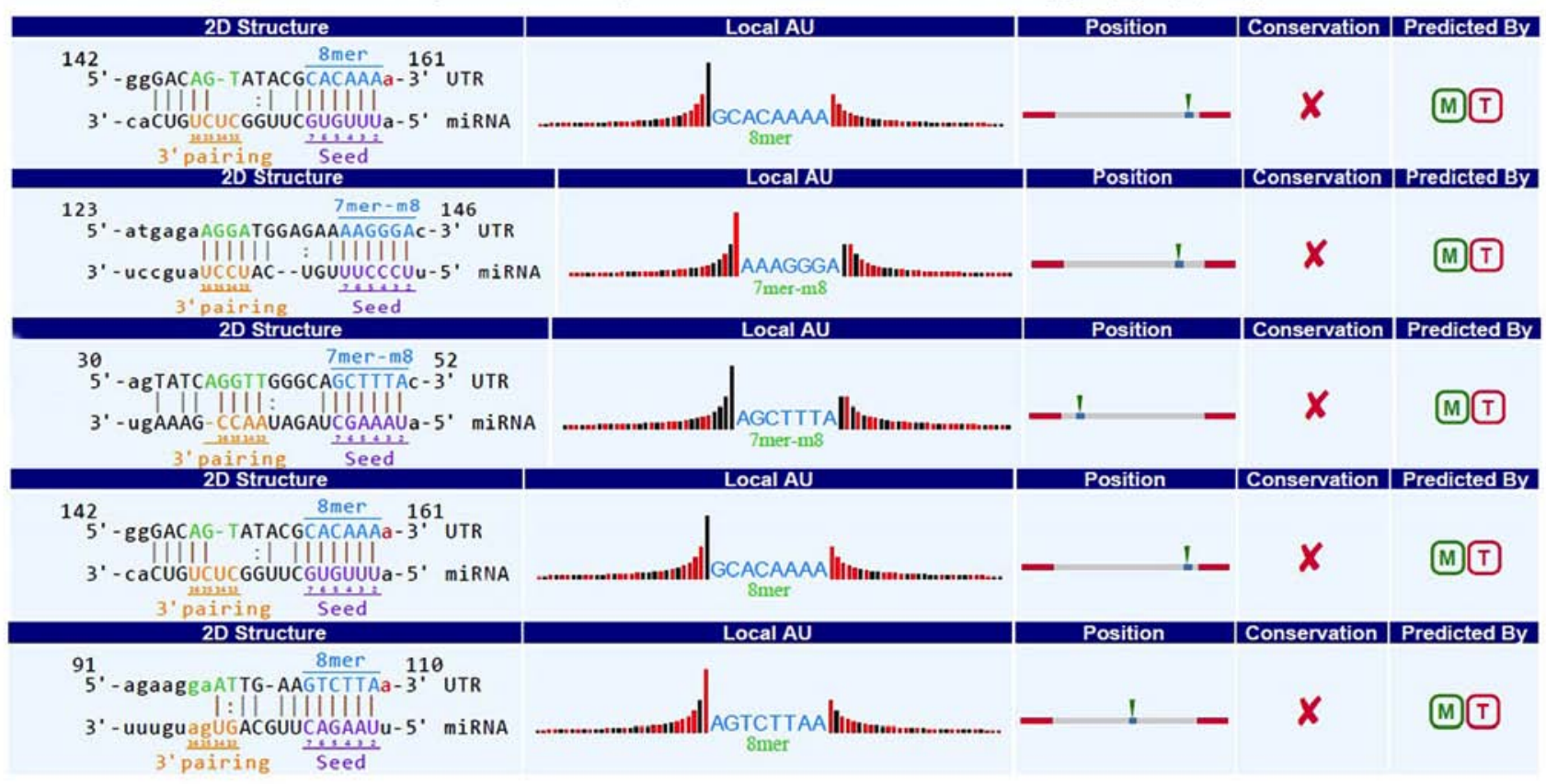

B hsa-miR-141-5p/hsa-miR-486-5p/hsa-miR-186-3p/hsa-miR-647/hsa-miR-212-5p_VS_hsa_circ_0067127

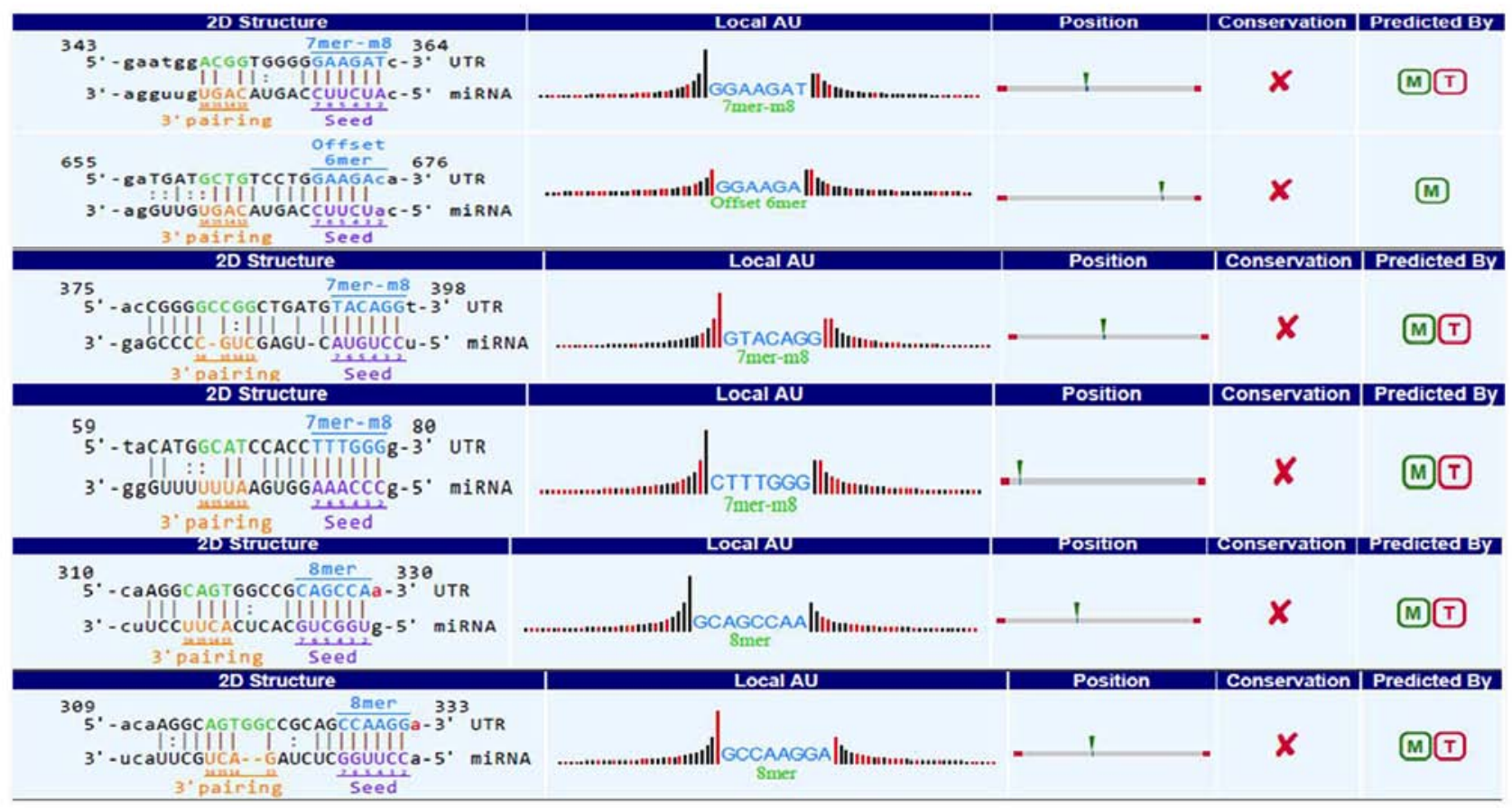

Figure 3. Virtual prediction of miRNAs targeting hsa_circ_0003056 and hsa_circ_0067127. Target miRNAs of circRNA were predicted by surveying for 7-mer or 8-mer complementarity to the seed region and the 3' pairing of each miRNA using TargetScan. The top five miRNAs were chosen based on the mirSVR scores for the establishment of ceRNA networks. (A) miRNAs targeting to hsa_circ_0003056; (B) miRNAs targeting to hsa_circ_0067127. miRNA, microRNA; circRNA, circular RNA; ceRNA, competing endogenous RNA.

network exhibited a strong association with 'artery morphogenesis activity', 'HOPS complex' and 'transferase activity, transferring acyl groups' in $\mathrm{BP}, \mathrm{CC}$ and $\mathrm{MF}$, respectively.

Cytoscape analysis of hsa_circ_0003056- and hsa circ_0067127-miRNA-targetgene interaction networks. Based on hsa_circ_0003056 and hsa_circ_0067127, the networks of five miRNAs with their target mRNAs were constructed. In these networks, green circular nodes represent mRNAs, pink diamond nodes represent miRNAs, gray lines represent those not experimentally validated, and blue lines represent those experimentally validated (miRTarBase 7.0.). Fig. 5 indicates the top five miRNAs targeting to hsa_circ_0003056 and their target mRNAs. The top three mRNAs corresponding to both hsa-miR-211-5p and hsa-miR-204-5p were adaptor related protein complex 1 subunit sigma 2 , solute carrier family 37 
A B
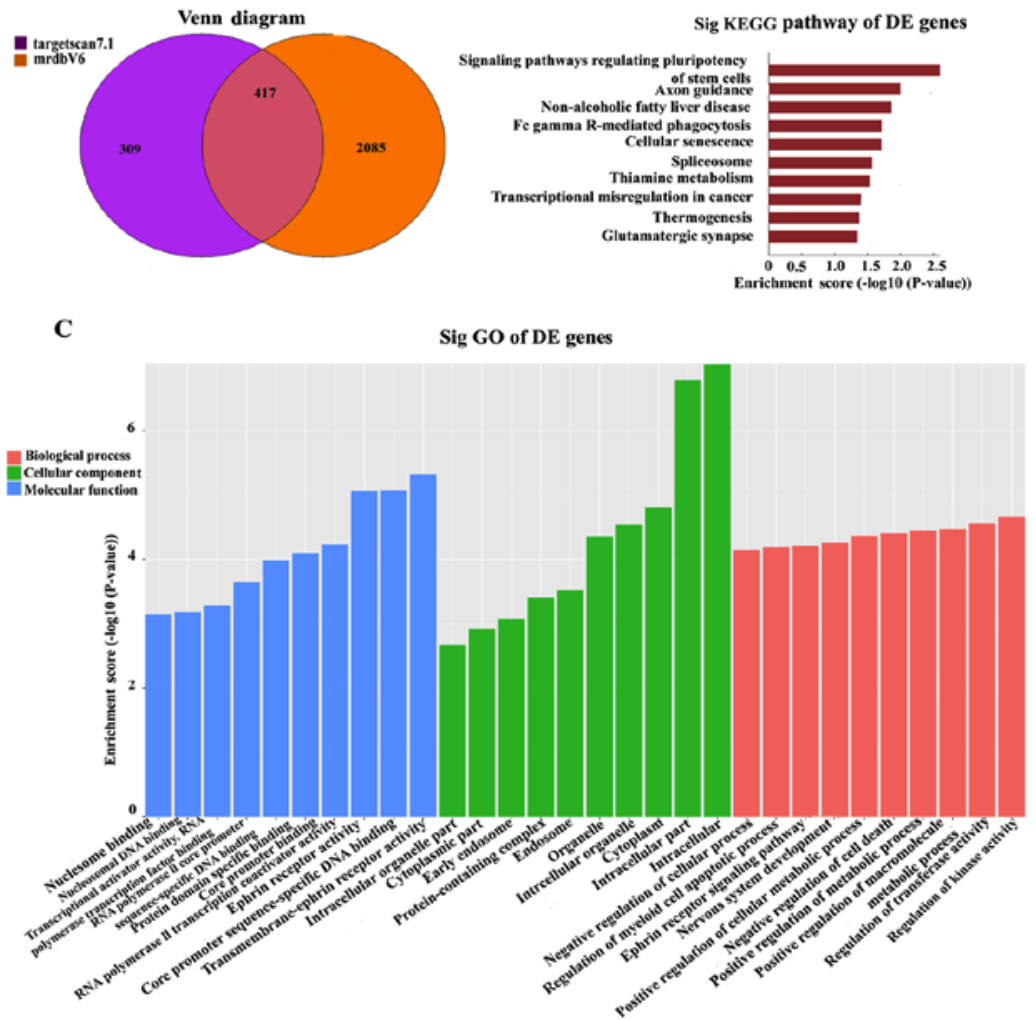

D

E
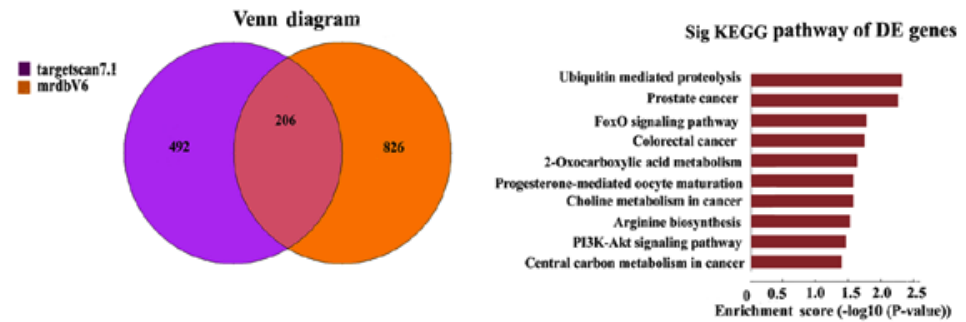

$\mathbf{F}$

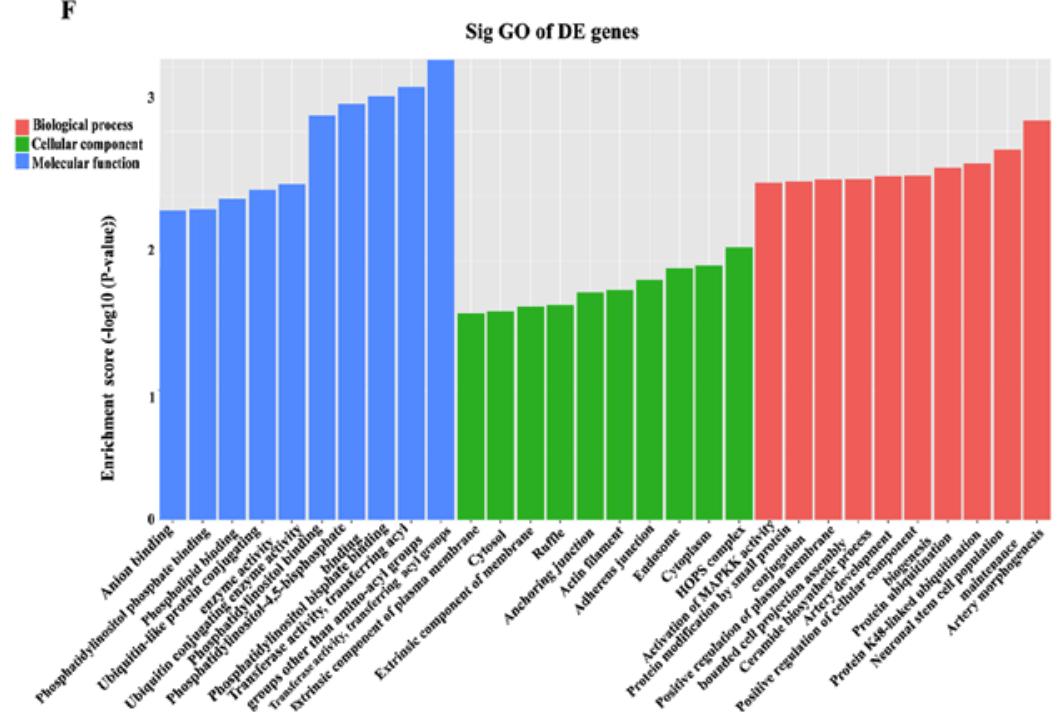

Figure 4. KEGG pathway and GO enrichment analyses based on the ceRNA network of hsa_circ_0003056 and hsa_circ_0067127. (A-C) Analyses based on the ceRNA network of hsa_circ_0003056. Venn diagram of the overlapping results of two databases: (A) TargetScan 7.1 and mirdbV5 were used for human genes to forecast the target genes of miRNAs; (B) The bar plot presents the enrichment scores [-log10 (P-value)] of the top 10 significant enrichment pathways of DE genes. GO analyses cover three domains; and (C) the bar plot shows the enrichment scores [-log10 (P-value)] of the top 10 significant GO enrichments. (D-F) Analyses based on the ceRNA network of hsa_circ_0067127: (D) Target genes of miRNAs predicted using TargetScan 7.1 and mirdbV5; (E) enrichment scores of the top 10 significant enrichment pathways of DE genes; and (F) top 10 significant GO enrichments. KEGG, Kyoto Encyclopedia of Genes and Genomes; GO, Gene Ontology; ceRNA, competing endogenous RNA; miRNA, microRNA; DE, differentially expressed. 


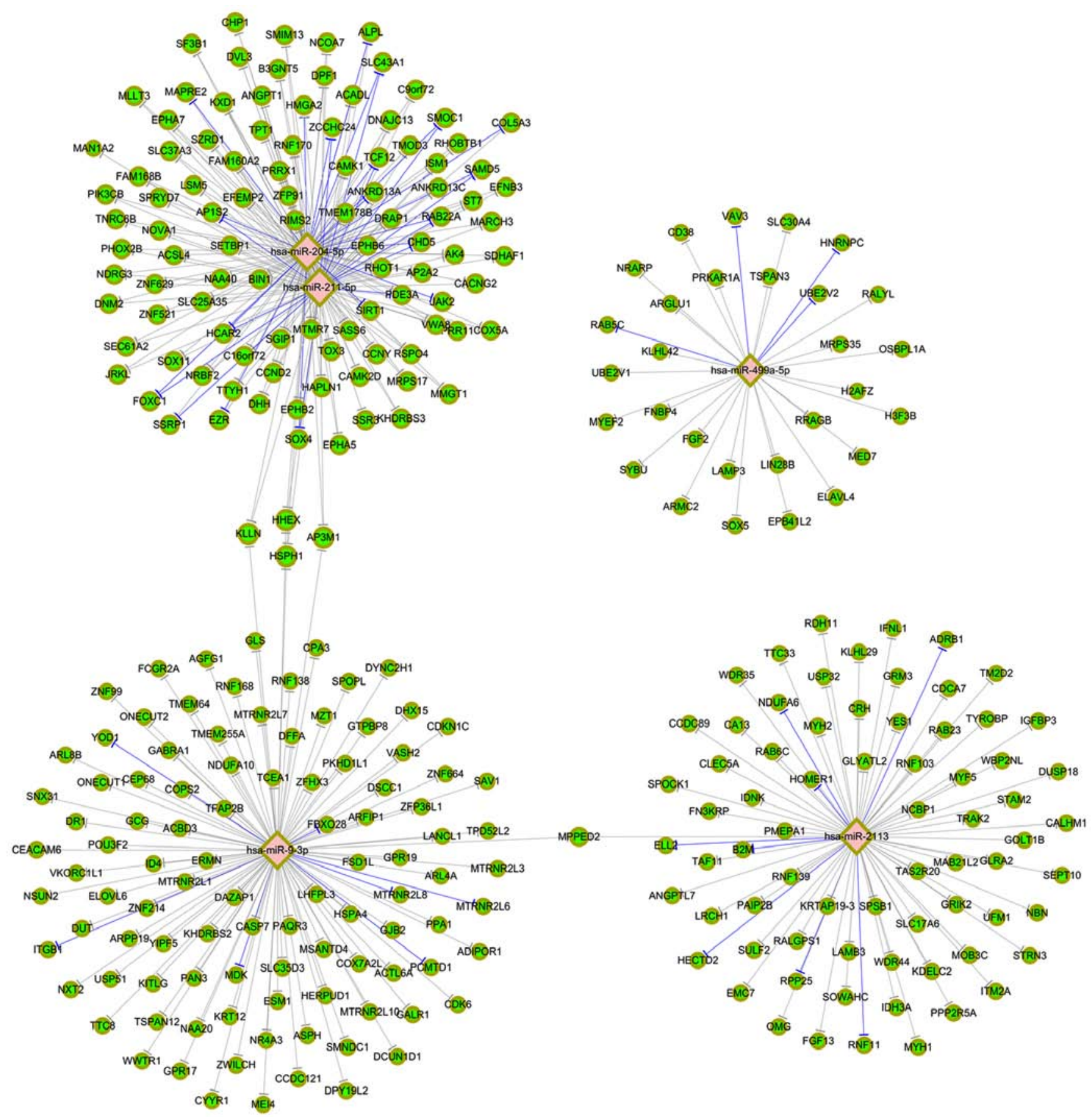

Figure 5. Cytoscape analysis of hsa_circ_0003056-miRNAs-target gene interaction networks. Networks of 5 miRNAs with their target mRNAs were constructed, in which green circular nodes represent mRNAs, pink diamond nodes represent miRNAs, gray lines represent not experimentally validated by miRTarBase 7.0, and blue lines represent experimentally validated by miRTarBase 7.0. miRNA, microRNA.

member 3 and RAB22A, member RAS oncogene family. Similarly, Fig. 6 presents the top five miRNAs targeting to hsa_circ_0067127 and their target mRNAs.

\section{Discussion}

It has been determined that circRNAs may influence the initiation and development of a range of different cancer types. Certain circRNAs are enriched in exosomes (16), suggesting that they exhibit the potential to function as tumor biomarkers, and may therefore be used to support diagnosis. However, few reports have investigated this with regards to PHC tissues and plasma. The current study provided a comprehensive circRNA profile in three pairs of PHC tissues and adjacent tissues prior to treatment, using circRNA chip screening. A total of five circRNAs were selected for validation of their respective expression levels in both PHC tissues, and adjacent tissues from 11 patients, using RT-qPCR analysis. However, only hsa_circ_0003056 was confirmed to be significantly downregulated in PHC tissues. According to the initial analyses of circRNA chip screening, the gene coding for hsa_circ_0003056 is located on chromosome 22, and the sequence of its best transcript is NM_012399, with a gene symbol of phosphatidylinositol (Ptdins) transfer protein 


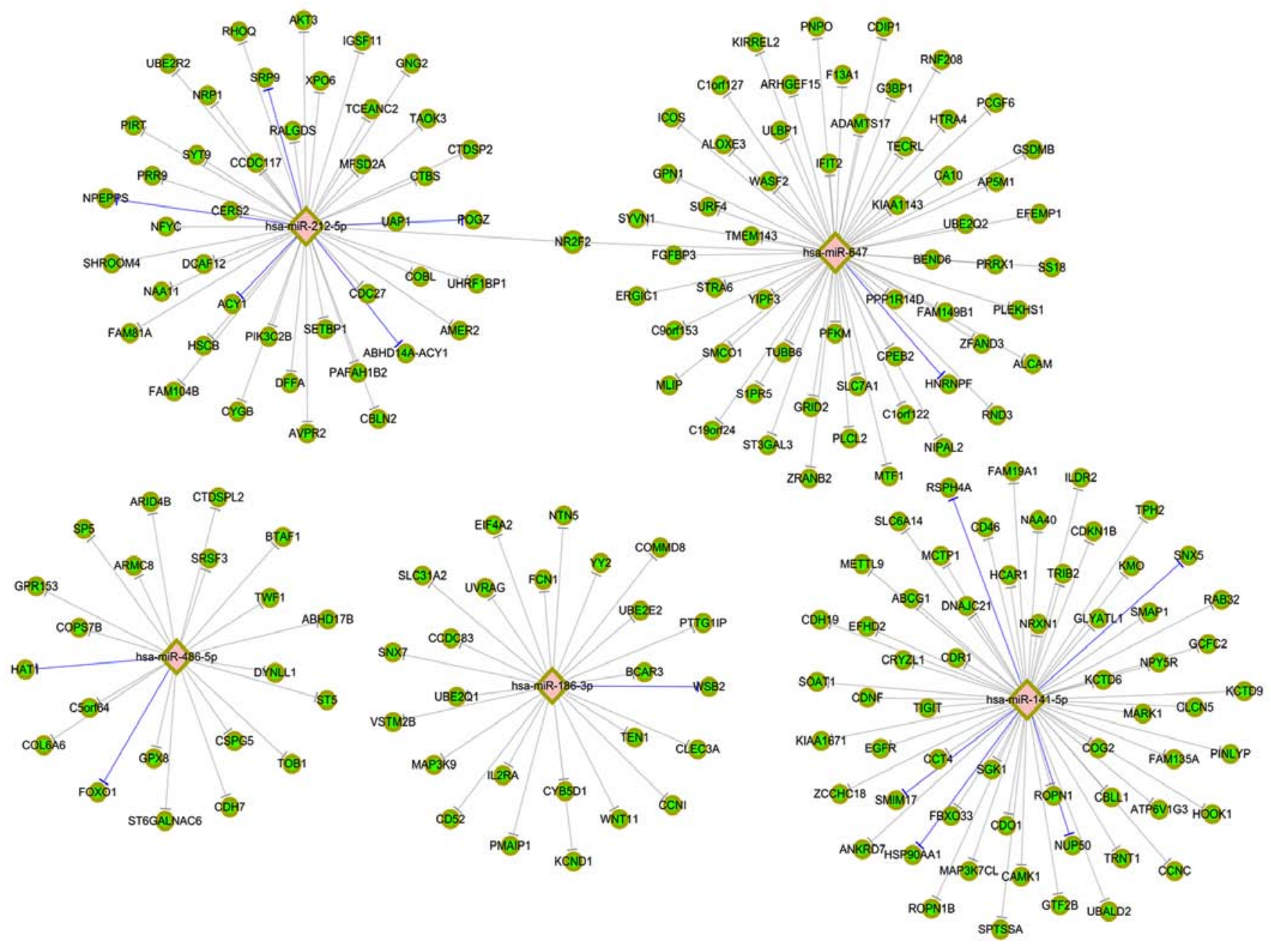

Figure 6. Cytoscape analyses of hsa_circ_0067127-miRNAs-target gene interaction networks. Networks of 5 miRNAs with their target mRNAs were constructed, in which green circular nodes represent mRNAs, pink diamond nodes represent miRNAs, gray lines represent not experimentally validated by miRTarBase7.0, blue lines represent experimentally validated by miRTarBase 7.0. miRNA, microRNA.

beta (PITPNB). The relatively linear PITPNB stimulates Ptdins-4-phosphate synthesis and signaling in eukaryotic cells (27). However, whether PITPNB influences tumor progression is yet to be determined. Therefore, the current study analyzed the associations between circPITPNB and certain clinicopathological parameters. However, the results revealed no significant association, which may be due to the small sample size of 11 cases. Further functional experiments are required to determine the mechanism behind the downregulation of hsa_circ_0003056 expression in PHC tissues.

Using RT-qPCR, hsa_circ_0003056 expression levels were determined in fresh plasma samples retrieved from 35 PHC patients and 32 healthy donors. The expression levels of hsa_circ_0003056 did not significantly decrease in the plasma taken from patients with PHC. This may be due to the fact that most of the plasma samples were collected from patients undergoing interventional therapy, and only a small number were subjected to specimen collection prior to treatment. It has been suggested that interventional therapies may influence the expression levels of hsa_circ_0003056 in the plasma. circRNAs are relatively stable in plasma, however, the total free nucleic acid content of the peripheral blood is low (28). There are no stable, internal or precise quantification methods for the determination of these levels. $\beta$-actin or GAPDH can be used in plasma or serum (29), but they are not ideal internal references due to their instability and high individual variation. Although circRNAs exhibit a degree of tissue specificity, they are expressed in plasma and can be detected. These circRNAs may represent novel potential serum biomarkers. For example, circRNA-284 and miR-221 both exhibit the potential to become diagnostic biomarkers of carotid plaque ruptures and strokes (30). In the present study, the expression level of hsa_circ_0067127 was lower in PHC plasma but not in PHC tissue (compared to normal plasma and para-tumor tissues, respectively), and was also associated with AFP level, as determined by stratified analysis. To further validate the expression levels of hsa_circ_0003056 and hsa_circ_0067127 in PHC plasma, the plasmid vector method should be used in future studies as a standard to quantify the two circRNAs, with an increased sample size for improved reliability.

Mechanisms describing the roles of circRNAs in cancer progression have not been clearly elucidated. The primary function of circRNAs is to act as miRNA sponges by forming circRNA-miRNA-mRNA axes (22). The circ_0067934/miR-1324/FZD5/b-catenin signaling complexes may serve as a promising therapeutic target for 
HCC intervention (31). Other functions of circRNAs, such as their interaction with RNA-binding proteins and translating proteins, have previously been described (32). In the present study, the top five target miRNAs that were indicated to interact with hsa_circ_0003056, were selected based on their mirSVR scores and TargetScan. These were hsa-miR-211-5p, hsa-miR-204-5p, hsa-miR-9-3p, hsa-miR-2113 and hsa-miR-499a-5p. Hsa-miR-211-5p levels in HCC tissues were lower than those in normal tissues, suggesting an inhibitory role in $\mathrm{HCC}$ by inhibiting zinc finger E-box-binding protein (ZEB2) expression (33). Hsa-miR-211-5p is also associated with renal cell carcinoma, thyroid tumors and triple-negative breast cancer (34-36). Hsa-miR-204-5p is associated with a variety of malignancies, including HCC and laryngeal squamous cell carcinoma $(37,38)$. Whether hsa-miR-204-5p is associated with $\mathrm{PHC}$ progression, through its interaction with hsa_circ_0003056, remains undetermined. A practical method is urgently required to identify specific interactions between miRNAs and circRNAs, currently identified using complicated bioinformatics prediction networks. KEGG pathway analyses revealed that DE genes were significantly enriched in pathways that regulates the pluripotency of stem cells. GO analyses revealed that hsa_circ_0003056 was strongly associated with 'regulation of kinase activity', and 'intracellular and transmembrane-ephrin receptor activity' in $\mathrm{BP}, \mathrm{CC}$ and $\mathrm{MF}$, respectively, which is consistent with its linear form (PITPNB), a gene encoding a cytoplasmic protein that catalyzes the transfer of phosphatidylinositol and phosphatidylcholine between membranes.

In the future, functional experiments should be performed to further explore the mechanism of circRNA in the regulation of PHC progression. Hepatic carcinoma cell lines with high or low expression levels of hsa_circ_0003056 should be constructed, and used to conduct proliferation and migration experiments. The binding and co-localization of hsa_circ_0003056 with the top five target miRNAs should be confirmed, and the target genes (mRNAs) corresponding to the miRNAs (as shown using GO enrichment analyses) should be quantified.

In summary, hsa_circ_0003056 expression is significantly decreased in PHC tissues, but is relatively stable in plasma. Conversely, hsa_circ_0067127 is downregulated in PHC plasma but not in PHC tissue. The detailed molecular mechanisms by which hsa_circ_0003056 and hsa_circ_0067127 function as miRNA sponges to regulate PHC occurrence and development require further investigation.

\section{Acknowledgements}

Not applicable.

\section{Funding}

The present study was supported by grants from the Natural Science Fund of Education Department of Anhui Province (grant no. KJ2016A380 and KJ2018A0797), the Science and Technology Project of Clinical Medicine Foundation of Jiangsu Province (grant no. BL2014005), the Key Development Project for Medical Science and Technology of Jiangsu Province (grant no. ZKX12038) and the Key
Project of Nanjing Health Bureau (grant no. 201208038). The sponsors had no role in the study design, data collection and analysis, preparation of the manuscript or the decision to submit the article for publication.

\section{Availability of data and materials}

The datasets used and/or analyzed during the present study are available from the corresponding author on reasonable request.

\section{Authors' contributions}

YY and CS designed and supervised the research. YD, AF and JY performed the experiments and data analyses. JD and NW collected the specimens. YD and CS wrote the manuscript. All authors read and approved the final manuscript.

\section{Ethics approval and consent to participate}

The present study was approved by the Ethics Committee of the Second Hospital of Nanjing (2017-LY-Kt007). Written informed consent was obtained from each patient and healthy donor enrolled. All procedures performed in the study involving human participants were in accordance with the ethical standards of the Institutional and/or National Research Committee and with the 1964 Helsinki declaration and its later amendments or comparable ethical standards.

\section{Patient consent for publication}

Not applicable.

\section{Competing interests}

The authors declare that they have no competing interests.

\section{References}

1. Ma L, Wang B, Long Y and Li H: Effect of traditional Chinese medicine combined with Western therapy on primary hepatic carcinoma: A systematic review with meta-analysis. Front Med 11: 191-202, 2017.

2. Chen W, Zheng R, Baade PD, Zhang S, Zeng H, Bray F, Jemal A $\mathrm{Yu}$ XQ and He J: Cancer statistics in China, 2015. CA Cancer J Clin 66: 115-132, 2016.

3. Wu X, Li J, Wang C, Zhang G, Zheng $\mathrm{N}$ and Wang X: Application of different imaging methods in the early diagnosis of primary hepatic carcinoma. Gastroenterol Res Pract 2016: 8763205, 2016.

4. Berretta M, Cavaliere C, Alessandrini L, Stanzione B, Facchini G, Balestreri L, Perin T and Canzonieri V: Serum and tissue markers in hepatocellular carcinoma and cholangiocarcinoma: Clinical and prognostic implications. Oncotarget 8: 14192-14220, 2017.

5. Peng L, Chen G, Zhu Z, Shen Z, Du C, Zang R, Su Y, Xie H, $\mathrm{Li} \mathrm{H}, \mathrm{Xu}$ X, et al: Circular RNA ZNF609 functions as a competitive endogenous RNA to regulate AKT3 expression by sponging miR-150-5p in Hirschsprung's disease. Oncotarget 8: 808-818, 2017.

6. Memczak S, Jens M, Elefsinioti A, Torti F, Krueger J, Rybak A, Maier L, Mackowiak SD, Gregersen LH, Munschauer M, et al: Circular RNAs are a large class of animal RNAs with regulatory potency. Nature 495: 333-338, 2013.

7. Salzman J: Circular RNA Expression: Its potential regulation and function. Trends Genet 32: 309-316, 2016.

8. Memczak S, Papavasileiou P, Peters O and Rajewsky N: Identification and characterization of circular RNAs as a new class of putative biomarkers in human blood. PLoS One 10: e141214, 2015. 
9. Li F, Zhang L, Li W, Deng J, Zheng J, An M, Lu J and Zhou Y: Circular RNA ITCH has inhibitory effect on ESCC by suppressing the $\mathrm{Wnt} / \beta$-catenin pathway. Oncotarget 6 : 6001-6013, 2015.

10. Liu Y, Cui H, Wang W, Li L, Wang Z, Yang S and Zhang X: Construction of circular miRNA sponges targeting miR-21 or miR-221 and demonstration of their excellent anticancer effects on malignant melanoma cells. Int J Biochem Cell Biol 45: 2643-2650, 2013.

11. Sand M, Bechara FG, Sand D, Gambichler T, Hahn SA, Bromba M, Stockfleth E and Hessam S: Circular RNA expression in basal cell carcinoma. Epigenomics 8: 619-632, 2016.

12. Zhang $\mathrm{C}$, Wu H, Wang Y, Zhao Y, Fang X, Chen $\mathrm{C}$ and Chen $\mathrm{H}$ : Expression patterns of circular RNAs from primary kinase transcripts in the mammary glands of lactating rats. J Breast Cancer 18: 235-241, 2015.

13. Bachmayr-Heyda A, Reiner AT, Auer K, Sukhbaatar N, Aust S, Bachleitner-Hofmann T, Mesteri I, Grunt TW, Zeillinger R and Pils D: Correlation of circular RNA abundance with proliferation-Exemplified with colorectal and ovarian cancer, idiopathic lung fibrosis, and normal human tissues. Sci Rep 5: 8057, 2015.

14. Li P, Chen S, Chen H, Mo X, Li T, Shao Y, Xiao B and Guo J: Using circular RNA as a novel type of biomarker in the screening of gastric cancer. Clin Chim Acta 444: 132-136, 2015

15. Qu S, Song W, Yang X, Wang J, Zhang R, Zhang Z, Zhang H and Li H: Microarray expression profile of circular RNAs in human pancreatic ductal adenocarcinoma. Genomics Data 5: 385-387, 2015.

16. Li Y, Zheng Q, Bao C, Li S, Guo W, Zhao J, Chen D, Gu J, He X and Huang S: Circular RNA is enriched and stable in exosomes: A promising biomarker for cancer diagnosis. Cell Res 25: 981-984, 2015.

17. Qin M, Liu G, Huo X, Tao X, Sun X, Ge Z, Yang J, Fan J, Liu L and Qin W: Hsa_circ_0001649: A circular RNA and potential novel biomarker for hepatocellular carcinoma. Cancer Biomark 16: 161-169, 2016.

18. Shang X, Li G, Liu H, Li T, Liu J, Zhao Q and Wang C: Comprehensive circular RNA profiling reveals that hsa_circ_0005075, a new circular RNA biomarker, is involved in hepatocellular carcinoma development. Medicine (Baltimore) 95: e3811, 2016.

19. Huang XY, Huang ZL, Xu YH, Zheng Q, Chen Z, Song W, Zhou J, Tang ZY and Huang XY: Comprehensive circular RNA profiling reveals the regulatory role of the circRNA-100338/miR-141-3p pathway in hepatitis B-related hepatocellular carcinoma. Sci Rep 7: 5428, 2017.

20. Fu L, Yao T, Chen Q, Mo X, Hu Y and Guo J: Screening differential circular RNA expression profiles reveals hsa_circ_0004018 is associated with hepatocellular carcinoma. Oncotarget 8: 58405-58416, 2017.

21. Yu J, Xu QG, Wang ZG, Yang Y, Zhang L, Ma JZ, Sun SH, Yang $F$ and Zhou WP: Circular RNA cSMARCA5 inhibits growth and metastasis in hepatocellular carcinoma. J Hepatol 68 : 1214-1227, 2018.

22. Hansen TB, Jensen TI, Clausen BH, Bramsen JB, Finsen B, Damgaard CK and Kjems J: Natural RNA circles function as efficient microRNA sponges. Nature 495: 384-388, 2013.

23. Enright AJ, John B, Gaul U, Tuschl T, Sander C and Marks DS: MicroRNA targets in Drosophila. Genome Biol 5: R1, 2003.
24. Pasquinelli AE: MicroRNAs and their targets: Recognition, regulation and an emerging reciprocal relationship. Nat Rev Genet 13: 271-282, 2012.

25. Huang da W, Sherman BT and Lempicki RA: Systematic and integrative analysis of large gene lists using DAVID bioinformatics resources. Nat Protoc 4: 44-57, 2009.

26. Livak KJ and Schmittgen TD: Analysis of relative gene expression data using real-time quantitative PCR and the 2(-Delta Delta C(T)) method. Methods 25: 402-408, 2001

27. Xie Z, Hur SK, Zhao L, Abrams CS and Bankaitis VA: A Golgi lipid signaling pathway controls apical Golgi distribution and cell polarity during Neurogenesis. Dev Cell 44: 725-740, 2018.

28. Sorber L, Zwaenepoel K, Jacobs J, De Winne K, Goethals S, Reclusa P, Van Casteren K, Augustus E, Lardon F, Roeyen G, et al: Circulating cell-free DNA and RNA analysis as liquid biopsy: Optimal centrifugation protocol. Cancers (Basel) 11: pii: E 458, 2019.

29. Lu R, Shao Y, Ye G, Xiao B and Guo J: Low expression of hsa_circ_0006633 in human gastric cancer and its clinical significances. Tumor Biol 39: 1-7, 2017.

30. Bazan HA, Hatfield SA, Brug A, Brooks AJ, Lightell DJ Jr and Woods TC: Carotid plaque rupture is accompanied by an increase in the ratio of serum circR-284 to miR-221 levels. Circ-Cardiovasc Gene 10: pii: e0017204, 2017.

31. Zhu Q, Lu G, Luo Z, Gui F, Wu J, Zhang D and Ni Y: CircRNA circ_0067934 promotes tumor growth and metastasis in hepatocellular carcinoma through regulation of miR-1324/FZD5/Wnt/ $\beta$-catenin axis. Biochem Bioph Res Co 497: 626-632, 2018.

32. Meng S, Zhou H, Feng Z, Xu Z, Tang Y, Li P and Wu M: CircRNA: Functions and properties of a novel potential biomarker for cancer. Mol Cancer 16: 94-102, 2017.

33. Jiang G, Wen L, Deng W, Jian Z and Zheng H: Regulatory role of miR-211-5p in hepatocellular carcinoma metastasis by targeting ZEB2. Biomed Pharmacother 90: 806-812, 2017.

34. Quan J, Pan X, He T, Lin C, Lai Y, Chen P, Zhang Z, Yang S, Wang T and Lai Y: Tumor suppressor miR-211-5p is associated with cellular migration, proliferation and apoptosis in renal cell carcinoma. Exp Ther Med 15: 4019-4028, 2018.

35. Chen LL, Zhang ZJ, Yi ZB and Li JJ: MicroRNA-211-5p suppresses tumour cell proliferation, invasion, migration and metastasis in triple-negative breast cancer by directly targeting SETBP1. Brit J Cancer 117: 78-88, 2017.

36. Wang L, Shen YF, Shi ZM, Shang XJ, Jin DL and Xi F: Overexpression miR-211-5p hinders the proliferation, migration, and invasion of thyroid tumor cells by downregulating SOX11. J Clin Lab Anal 32: 2018.

37. Jiang G, Wen L, Zheng H, Jian $Z$ and Deng W: miR-204-5p targeting SIRT1 regulates hepatocellular carcinoma progression. Cell Biochem Funct 34: 505-510, 2016.

38. Gao W, Wu Y, He X, Zhang C, Zhu M, Chen B, Liu Q, Qu X, Li W, Wen S and Wang B: MicroRNA-204-5p inhibits invasion and metastasis of laryngeal squamous cell carcinoma by suppressing forkhead box C1. J Cancer 8: 2356-2368, 2017.

This work is licensed under a Creative Commons Attribution-NonCommercial-NoDerivatives 4.0 International (CC BY-NC-ND 4.0) License. 